\title{
Allgemeines Literaturverzeichnis
}

Adler/Düring/Schmaltz, Rechnungslegung und Prüfung der Unternehmen, 6. Aufl. $1994 \mathrm{ff}$.

Baetge/Kirsch/Thiele, Bilanzrecht (Loseblatt)

Baumbach/Hopt, Handelsgesetzbuch, 38. Aufl. 2018

Baumbach/Hueck, GmbH-Gesetz, 21. Aufl. 2017

Baur/Stürner/Bruns, Zwangsvollstreckungs-, Konkurs- und Vergleichsrecht, Band II, Insolvenzrecht, 13. Aufl. 2006

Beck'scher Bilanz-Kommentar, Handels- und Steuerbilanz - $\$ \$ 238-339,342-342$ e HGB, 11. Aufl. 2018

Beck'sches Handbuch der GmbH, herausgegeben von Ulrich Prinz und Norbert Winkeljohann, 5. Aufl. 2014

Beck'sches Handbuch der Personengesellschaften, herausgegeben von Welf Müller, Wolf-

Dieter Hoffmann und Ulrich Prinz, 4. Aufl. 2014

Biergans, Einkommensteuer und Steuerbilanz, 6. Aufl. 1992

Binz, Haftungsverhältnisse im Gründungsstadium der GmbH \& Co. KG, 1976

Binz/Sorg, Die GmbH \& Co. KG, 12. Aufl. 2018

Birkenfeld/Wäger, Das große Umsatzsteuer-Handbuch (Loseblatt)

Blaurock, Handbuch Stille Gesellschaft, 8. Aufl. 2016

Blümich, Einkommensteuergesetz, Körperschaftsteuergesetz, Gewerbesteuergesetz (Loseblatt)

Boesebeck, Die kapitalistische Kommanditgesellschaft, 1938

Bordewin/Brandt, Kommentar zum Einkommensteuergesetz (Loseblatt)

Boruttau, Grunderwerbsteuergesetz, 19. Aufl. 2019

Brandmüller, Betriebsaufspaltung (Loseblatt)

Brandmüller, Die Betriebsaufspaltung nach Handels- und Steuerrecht, einschließlich Betriebsverpachtung, 7. Aufl. 1997

Braun (Hrsg.), Insolvenzordnung, 7. Aufl. 2017

Brönner, Die Besteuerung der Gesellschaften, 18. Aufl. 2007

Cahn-Garnier, GmbH \& Co., Kommanditgesellschaft, 1922

Carlé, Dieter/Carlé, Thomas/Bauschatz, Peter, Die Betriebsaufspaltung, 2. Aufl. 2014

Crezelius, Unternehmenserbrecht, 2. Aufl. 2009

Daeschner, Handelsgesellschaftliche Gestaltungsformen, 1923

Dankmeyer/Giloy, Einkommensteuergesetz (Loseblatt)

Dietrich, Die Publikums-Kommanditgesellschaft und die gesellschaftsrechtlich geschützten Interessen, 1988

Dötsch/Patt/Pung/Möhlenbrock, Umwandlungssteuerrecht, 7. Aufl. 2012

Dötsch/Pung/Möhlenbrock, Die Körperschaftsteuer (Loseblatt)

Düringer/Hachenburg/Flechtheim, Das Handelsgesetzbuch, 3. Aufl. 1923

Ebenroth, Die Kontrollrechte der GmbH-Gesellschafter, 1971

Ebenroth/Boujong/Joost/Strohn, HGB, Kommentar, Bd. 1: \$\$1-342e, 3. Aufl. 2014

Eickhoff, Die Praxis der Gesellschafterversammlung, 4. Aufl. 2006

Eltermann, Der verdeckte Kapitalentzug in der GmbH \& Co. KG, Diss., 1972 
Erman, BGB, Handkommentar, herausgegeben von Barbara Grunewald, Georg MaierReimer und Harm Peter Westermann, 15. Aufl. 2017

FGS/BDI, Der Umwandlungssteuer-Erlass 2011, herausgegeben von Flick Gocke Schaumburg und dem Bundesverband der Deutschen Industrie e.V., 2012

Flume, Allgemeiner Teil des Bürgerlichen Rechts, Bd. 1, 1. Teil: Die Personengesellschaft, 1977; Bd. 1, 2. Teil: Die juristische Person, 1983

Frankfurter Kommentar zur Insolvenzordnung, herausgegeben von Klaus Wimmer, 9. Aufl. 2018

Frotscher/Geurts, Kommentar zum Einkommensteuergesetz (Loseblatt)

Frotscher/Maas, Kommentar zum Körperschaft-, Gewerbe- und Umwandlungssteuergesetz (Loseblatt)

Gierke, J.v., Handelsrecht und Schiffahrtsrecht, 8. Aufl. 1958

Glade/Steinfeld, Kommentar zum Umwandlungssteuergesetz 1977, 3. Aufl. 1980

Glanegger/Güroff, Gewerbesteuergesetz, 9. Aufl. 2017

$\mathrm{GmbH}-\mathrm{Handbuch}$, herausgegeben von der Centrale für $\mathrm{GmbH}$, bearbeitet von Bochmann, Brand, Cziupka, Fuhrmann, Honert, Neu, Neumann, Neumayer, Reufels, Rodewald, Schiffers, Theile, Wälzholz (Loseblatt)

Großkommentar HGB, herausgegeben von Claus-Wilhelm Canaris, Mathias Habersack, Carsten Schäfer, 5. Aufl. $2009 \mathrm{ff}$.

Haritz/Menner/Bilitewski, Umwandlungssteuergesetz, 5. Aufl. 2019

Heidelberger Kommentar zum HGB, herausgegeben von Peter Glanegger, Christian Kirnberger, Stefan Kusterer u.a., 7. Aufl. 2007

Heidelberger Kommentar zum KStG, herausgegeben von Bernd Erle, Thomas Sauter, 3. Aufl. 2010

Herrmann/Heuer/Raupach, Einkommensteuergesetz und Körperschaftsteuergesetz (Loseblatt)

Heymann, Handelsgesetzbuch, Erstes, Drittes und Viertes Buch 2. Aufl. 2012 ff.; Zweites Buch 3. Aufl. 2017

Hofbauer/Kupsch, Rechnungslegung (Loseblatt)

Hofmann, Grunderwerbsteuergesetz, 11. Aufl. 2016

Huber, Vermögensanteil, Kapitalanteil und Gesellschaftsanteil an Personengesellschaften des Handelsrechts, 1970

Hübschmann/Hepp/Spitaler, Abgabenordnung - Finanzgerichtsordnung (Loseblatt)

Hüffer/Koch, Aktiengesetz, 13. Aufl. 2018

Jacobs/Scheffler/Spengel, Unternehmensbesteuerung und Rechtsform, 5. Aufl. 2015

Jaeger, Der Anstellungsvertrag des GmbH-Geschäftsführers, 6. Aufl. 2016

John, Die organisierte Rechtsperson, 1977

Kaligin, Die Betriebsaufspaltung, 11. Aufl. 2019

Kallmeyer, Umwandlungsgesetz, 6. Aufl. 2017

Kapp/Ebeling, Erbschaftsteuer- und Schenkungsteuergesetz (Loseblatt)

Kirchhof, EStG, Kommentar, 18. Aufl. 2019

Kirchhof/Söhn/Mellinghoff, Einkommensteuergesetz (Loseblatt)

Klein/Müller, Praxishandbuch der GmbH, 4. Aufl. 2017

Knobbe-Keuk, Bilanz- und Unternehmenssteuerrecht, 9. Aufl. 1993 
Koller/Kindler/Roth/Drüen, Handelsgesetzbuch, 9. Aufl. 2019

Korn/Carlé/Stahl, Einkommensteuergesetz (Loseblatt)

Köster, Anfechtungs- und Nichtigkeitsklage gegen Gesellschafterbeschlüsse bei OHG und KG, 1981

Kübler/Prütting/Bork, Kommentar zur Insolvenzordnung (Loseblatt)

Küting/Pfitzer/Weber (Hrsg.), Handbuch der Rechnungslegung - Einzelabschluss (Loseblatt)

Küting/Weber (Hrsg.), Handbuch der Konzernrechnungslegung, 2. Aufl. 1998

Lademann/Söffing/Brockhoff, Kommentar zum Einkommensteuergesetz (Loseblatt)

Lenski/Steinberg, Kommentar zum Gewerbesteuergesetz (Loseblatt)

Littmann/Bitz/Pust, Das Einkommensteuerrecht (Loseblatt)

Lutter, Umwandlungsgesetz, 6. Aufl. 2019

Lutter (Hrsg.), Die Gründung einer Tochtergesellschaft im Ausland, 3. Aufl. 1995

Lutter/Hommelhoff, GmbH-Gesetz, 19. Aufl. 2016

Meilicke/Meilicke, Kommentar zum Mitbestimmungsgesetz 1976, 2. Aufl. 1976

Meincke/Hannes/Holtz, Erbschaftsteuer- und Schenkungsteuergesetz, 17. Aufl. 2018

Michalski/Heidinger/Leible/J. Schmidt, Kommentar zum Gesetz betreffend die Gesellschaften mit beschränkter Haftung, (GmbH-Gesetz), 3. Aufl. 2017

Moench/Weinmann, Erbschaft- und Schenkungsteuer (Loseblatt)

Müller/Stöcker/Lieber, Die Organschaft, 10. Aufl. 2017

Münchener Handbuch des Gesellschaftsrechts, Bd. 1, BGB-Gesellschaft, Offene Handelsgesellschaft, Partnerschaftsgesellschaft, Partnerreederei, EWIV, herausgegeben von Hans Gummert und Lutz Weipert, 5. Aufl. 2019; Bd. 2, Kommanditgesellschaft, GmbH \& Co. KG, Publikums-KG, Stille Gesellschaft, herausgegeben von Hans Gummert und Lutz Weipert, 5. Aufl. 2019; Bd. 3, Gesellschaft mit beschränkter Haftung, herausgegeben von Hans-Joachim Priester, Dieter Mayer und Hartmut Wicke, 5. Aufl. 2018

Münchener Kommentar zum BGB, herausgegeben von Franz Jürgen Säcker, Roland Rixecker, Hartmut Oetker und Bettina Limperg, 8. Aufl. $2018 \mathrm{ff}$.

Münchener Kommentar zum GmbHG, herausgegeben von Holger Fleischer und Wulf Goette, 3. Aufl. $2018 \mathrm{f}$.

Münchener Kommentar zum HGB, herausgegeben von Karsten Schmidt, 3. Aufl. 2010 ff.; 4. Aufl. $2016 \mathrm{ff}$.

Münchener Kommentar zur InsO, herausgegeben von Rolf Stürner, Horst Eidenmüller und Heinrich Schoppmeyer, 4. Aufl. $2019 \mathrm{ff}$.

Münchener Vertragshandbuch, Bd. 1 Gesellschaftsrecht, herausgegeben von Nicolas Böhm und Frank Burmeister, 8. Aufl. 2018

Nieder/Kössinger, Handbuch der Testamentsgestaltung, 5. Aufl. 2015

Pahlke, Grunderwerbsteuergesetz, 6. Aufl. 2018

Palandt, Kommentar zum Bürgerlichen Gesetzbuch, 78. Aufl. 2019

Paulick, Das Recht der eingetragenen Genossenschaft, 1956

Raiser/Veil/Jacobs, Mitbestimmungsgesetz und Drittelbeteiligungsgesetz, 6. Aufl. 2015

Rau/Dürrwächter, Kommentar zum Umsatzsteuergesetz (Loseblatt)

Reichert, GmbH \& Co. KG, 7. Aufl. 2015 (bis 6. Aufl. herausgegeben von Heinrich Sudhoff) Reiß/Kraeusel/Langer, Umsatzsteuergesetz (Loseblatt) 
Reul/Heckschen/Wienberg, Insolvenzrecht in der Gestaltungspraxis, 2. Aufl. 2018

Reuter, Privatrechtliche Schranken der Pepetuierung von Unternehmen, 1973

Rödder/Herlinghaus/van Lishaut, Umwandlungssteuergesetz, 3. Aufl. 2019

Rödder/Hötzel/Mueller-Thuns, Unternehmenskauf, Unternehmensverkauf, 2003

Röhricht/Graf von Westphalen/Haas (Hrsg.), Handelsgesetzbuch, 5. Aufl. 2019

Roth/Altmeppen, Gesetz betreffend die Gesellschaften mit beschränkter Haftung, 9. Aufl. 2019

Rowedder/Schmidt-Leithoff (Hrsg.), Gesetz betreffend die Gesellschaften mit beschränkter Haftung, 6. Aufl. 2017

Sagasser/Bula/Brünger, Umwandlungen, 5. Aufl. 2017

Sassenrath, Die Umwandlung von Komplementär- in Kommanditbeteiligungen, 1988

Schaumburg/Rödder, Unternehmenssteuerreform 2001, 2000

Schaumburg/Rödder, Unternehmensteuerreform 2008

Schimansky/Bunte/Lwowski, Bankrechts-Handbuch, 5. Aufl. 2017

Schlegelberger, Handelsgesetzbuch, 5. Aufl. 1973-1992

Schmidt, Hans Martin (Hrsg.), Pro GmbH, Analysen und Perspektiven des Gesellschaftsund Steuerrechts der GmbH, 1980

Schmidt, Karsten, Gesellschaftsrecht, 4. Aufl. 2002

Schmidt, Karsten, Informationsrechte in Gesellschaften und Verbänden, 1974

Schmidt, Karsten, Liquidationsbilanzen und Konkursbilanzen, 1989

Schmidt, Karsten/Uhlenbruck, Wilhelm (Hrsg.), Die GmbH in Krise, Sanierung und Insolvenz, 5. Aufl. 2016

Schmidt, Ludwig, Einkommensteuergesetz, 38. Aufl. 2019

Schmitt/Hörtnagl/Stratz, Umwandlungsgesetz, Umwandlungssteuergesetz, 8. Aufl. 2018

Scholz, Kommentar zum GmbH-Gesetz, Bd. I: $₫ \$ 1-34$, 12. Aufl. 2018; Bd. II: $\$ \$ 35-52$,

11. Aufl. 2014; Bd. III: $\$ \$ 45-87,11$. Aufl. 2015

Schoor/Natschke, Die GmbH \& Still im Steuerrecht, 4. Aufl. 2005

Schulze zur Wiesche/Ottersbach, GmbH \& Co. KG, 3. Aufl. 2005

Semler/Stengel (Hrsg.), Umwandlungsgesetz, 4. Aufl. 2017

Soergel, Kommentar zum BGB, 13. Aufl. 1999 ff.

Söffing/Micker, Die Betriebsaufspaltung, 7. Aufl. 2018

Sölch/Ringleb, Umsatzsteuergesetz (Loseblatt)

Staub, Handelsgesetzbuch Großkommentar, 5. Aufl. 2009 ff.

Streck, Körperschaftsteuergesetz, 9. Aufl. 2018

Sudhoff, Heinrich, Unternehmensnachfolge, 5. Aufl. 2005

Sudhoff, Heinrich/Sudhoff, Martin, Rechte und Pflichten des Geschäftsführers einer GmbH, 14. Aufl. 1994

Tillmann/Mohr, GmbH-Geschäftsführer, 10. Aufl. 2013

Tipke/Kruse, Abgabenordnung. Finanzgerichtsordnung (Loseblatt)

Tipke/Lang, Steuerrecht, 23. Aufl. 2018

Troll/Gebel/Jülicher, Erbschaftsteuer- und Schenkungsteuergesetz (Loseblatt)

Uhlenbruck, Die GmbH \& Co. KG in Krise, Konkurs und Vergleich, 2. Aufl. 1988

Uhlenbruck, Insolvenzordnung, Bd. I: 15. Aufl. 2019; Bd. II 14. Aufl. 2015

Ulmer/Habersack/Löbbe, GmbHG, 2. Aufl. $2013 \mathrm{ff}$.

Viskorf/Schuck/Wälzholz, Erbschaftsteuer- und Schenkungsteuergesetz, Bewertungsgesetz, 5. Aufl. 2017 
von Oertzen/Loose, Erbschaftsteuer- und Schenkungsteuergesetz, 2017

Wagner/Rux, Die GmbH \& Co. KG, 12. Aufl. 2013

Weitnauer/Boxberger/Anders, KAGB, Kommentar zum Kapitalanlagebesetzbuch und zur Verordnung über Europäische Risikokapitalfonds mit Bezügen zum AIFM-StAnpG, 2. Aufl. 2017

Westermann, Harm Peter, Die GmbH \& Co. KG im Lichte der Wirtschaftsverfassung, 1973 Westermann/Wertenbruch, Handbuch Personengesellschaften (Loseblatt)

Wicke, Gesetz betreffend die Gesellschaften mit beschränkter Haftung (GmbHG), 3. Aufl. 2016

Widmann/Mayer, Umwandlungsrecht (Loseblatt)

Wiedmann/Böcking/Gros, Bilanzrecht, Kommentar zu den $\$ \$ 238-342$ e HGB, 3. Aufl. 2014

Wiethölter, Die GmbH \& Co. KG - Chancen und Grenzen, in: Aktuelle Probleme der GmbH \& Co., 3. Aufl. 1974

Willemsen/Hohenstatt/Schweibert/Seibt, Umstrukturierung und Übertragung von Unternehmen, 5. Aufl. 2016

Wilms/Jochum, Erbschaft- und Schenkungsteuergesetz (Loseblatt)

Winkeljohann/Förschle/Deubert, Sonderbilanzen, 5. Aufl. 2016

Winnefeld, Bilanz-Handbuch, 5. Aufl. 2015

Zimmermann/Hottmann/Kiebele/Schaeberle/Scheel/Schustek/Szczesny, Die Personengesellschaft im Steuerrecht, 12. Aufl. 2017 
